\title{
ON THE MAXIMAL MONOTONICITY AND THE RANGE OF THE SUM OF NONLINEAR MAXIMAL MONOTONE OPERATORS
}

\author{
by J. R. L. WEBB and WEIYU ZHAO
}

(Received 3rd July 1989)

\begin{abstract}
Conditions are given on two maximal monotone (multivalued) operators $A$ and $B$ which ensure that $A+B$ is also maximal. One condition used is that $\|B x\| \leqq k(\|x\|)|A x|+d|(A+B) x|+c(\|x\|)$ for every $x \in D(A) \subseteq D(B)$, where $0 \leqq k(r)<1$, and $c(r) \geqq 0$ are nondecreasing functions, and $0 \leqq d<1$ is a constant. Here, for a set $C$, $|C|$ denotes inf $\{\|y\|: y \in C\}$. This extends the well known result which has $d=0$ (and is used in the proof here). The second part of the paper uses similar hypotheses to give conditions under which the range of tine sum, $R(A+B)$, has the same interior and same closure as the sum of the ranges, $R(A)+R(B)$.
\end{abstract}

1980 Mathematics subject classification (1985 Revision): 47H05, secondary 47H06, 47H20.

\section{Introduction}

In this paper we study the maximal monotonicity and the range of the sum of two maximal monotone operators. Throughout this paper, $X$ is a real Banach space with dual space $X^{*}$, and the value of $x^{*} \in X^{*}$ at $x \in X$ is denoted by $\left(x, x^{*}\right)$. The norms in $X$ and $X^{*}$ are denoted by \|\| . A multivalued operator $A: X \rightarrow X^{*}$ is identified with its graph as a subset of $X \times X^{*}$, with its domain and range denoted by $D(A)$ and $R(A)$ respectively. $A$ is said to be monotone, if for each $\left(x_{i}, x_{i}^{*}\right) \in A(i=1,2)$ we have $\left(x_{1}-x_{2}, x_{1}^{*}-x_{2}^{*}\right) \geqq 0$. A monotone operator is said to be maximal monotone if it cannot be properly extended, in the sense of graph inclusion, to any other monotone operator.

If $A$ and $B$ are two monotone operators from $X$ to $X^{*}, A+B$ is clearly monotone. However, when both $A$ and $B$ are maximal monotone, $A+B$ is not necessarily maximal. So it is worthwhile to consider under what useful conditions the sum of two maximal monotone operators is maximal monotone. This problem is of importance in the existence theory for nonlinear partial differential equations. Some results in this direction were established in Attouch [1], Brezis-Crandall-Pazy [3], Browder [5], Calvert [6], Kato [8] and Rockafellar [10].

The basic condition given in [3, Theorem 2.3] is:

$$
|B x| \leqq k(\|x\|)|A x|+c(\|x\|) \text {, for every } x \in D(A) \subseteq D(B)
$$

where $0 \leqq k(r)<1$ and $c(r) \geqq 0$ are nondecreasing functions of $r$. Here for any subset 
$C \neq \theta$ of $X$ (or $X^{*}$ ), $|C|$ denotes $\inf \{\|x\|: x \in C\}$. In this paper we give a result under the following condition (we suppose $B$ is single-valued):

$$
\|B x\| \leqq k(\|x\|)|A x|+d|(A+B) x|+c(\|x\|), \text { for every } x \in D(A) \subseteq D(B)
$$

where $k(r)$ and $c(r)$ are as above, $0 \leqq d<1$ is a constant. This extends the result in [3] mentioned above. Note that this condition automatically holds with $k(\|x\|)=1$ and $d=1$. The other condition we give involves the duality mapping $F: X \rightarrow X^{*}$ defined by:

$$
F(x)=\left\{x^{*} \in X^{*}:\left(x, x^{*}\right)=\|x\|^{2}=\left\|x^{*}\right\|^{2}\right\} .
$$

$F$ is monotone and maximal monotonicity of a monotone operator $A$ can be determined using $F$. We assume that

$$
\begin{gathered}
\left(F^{-1} B x, x^{*}\right) \geqq-k(\|x\|)\left\|x^{*}\right\|^{2}-c\left(\left\|x^{*}\right\|\right)-h(\|x\|) p\left(\left\|x^{*}\right\|\right), \\
\text { for every } x \in D(A) \subseteq D(B), x^{*} \in A x
\end{gathered}
$$

where $0 \leqq k(r)<1, c(r) \geqq 0, h(r) \geqq 0$, and $p(r) \geqq 0$ are nondecreasing functions of $r$, and

$$
\frac{c(r)}{r^{2}} \rightarrow 0(r \rightarrow \infty), \frac{p(r)}{r} \text { is bounded when } r \rightarrow \infty .
$$

This looks similar to the condition given in Theorem 1 of [6], but is, in fact, quite different. It seems that our results cannot be proved using only the methods of proof in [3] and [6], nor can the results of [6] be obtained from ours. Moreover, we also give conditions of the same type under which $R(A+B)$ is almost equal to $R(A)+R(B)$ in that these sets have the same interiors and the same closures, using ideas similar to that of Pazy [4, Appendix] and Reich [9].

\section{Results}

\subsection{Perturbations of maximal monotone operators}

Let us recall the following results which may be found in Barbu [2, Section 1 of Chapter 2], Brezis-Crandall-Pazy [3] and Browder [5]. Let $X$ and $X^{*}$ be reflexive and strictly convex, then a monotone operator $B: X \rightarrow X^{*}$ is maximal monotone if, and only if, $R(F+B)=X^{*}$. If $B$ is maximal monotone, the equation

$$
F\left(x_{\lambda}-x\right)+\lambda x_{\lambda}^{*}=0, \quad x_{\lambda} \in D(B), \quad x_{\lambda}^{*} \in B x_{\lambda},
$$

has a unique solution $\left(x_{\lambda}, x_{\lambda}^{*}\right)$ for every $x \in X$ and $\lambda>0$. We define $J_{\lambda}$ and $B_{\lambda}$ by $J_{\lambda} x=x_{\lambda}, B_{\lambda} x=x_{\lambda}^{*}(x \in X)$ respectively. It is well-known that $B_{\lambda} x_{\lambda} \in B J_{\lambda} x_{\lambda}$. If $A: X \rightarrow X^{*}$ is another maximal monotone operator, $A+B_{\lambda}$ is maximal monotone. 
The following proposition gives our first result, which is then used to derive our main theorems.

Proposition 1. Let $X$ be a reflexive Banach space, let $A$ and $B$ be maximal monotone operators from $X$ to $X^{*}$, with $B$ single-valued and with $D(A) \subseteq D(B)$. If either

$$
\|B x\| \leqq a\left\|x^{*}\right\|+b\left\|x^{*}+B x\right\|+c(\|x\|), \text { for every } x \in D(A), x^{*} \in A x
$$

or

$$
\|B x\| \leqq a|A x|+b|(A+B) x|+c(\|x\|), \text { for every } x \in D(A)
$$

holds, where $0 \leqq a<1,0 \leqq b<1$ are constants, and $c(r) \geqq 0$ is a nondecreasing function of $r$, then $A+B$ is maximal monotone.

Proof. Obviously, inequality (1) holds whenever inequality (2) holds. It is therefore sufficient to prove the proposition under condition (1). For every $0 \leqq \alpha \leqq 1, x \in D(A)$, and $x^{*} \in A x$, from (1) we have

$$
\begin{aligned}
\|B x\| & \leqq a\left\|x^{*}+\alpha B x\right\|+a \alpha\|B x\|+b\left\|x^{*}+\alpha B x\right\|+b(1-\alpha)\|B x\|+c(\|x\|) \\
& \leqq(a+b)\left\|x^{*}+\alpha B x\right\|+d\|B x\|+c(\|x\|)
\end{aligned}
$$

where $d=\max (a, b)$. Therefore

$$
\|B x\| \leqq \frac{a+b}{1-d}\left\|x^{*}+\alpha B x\right\|+\frac{1}{1-d} c(\|x\|) .
$$

Hence

$$
\|B x\| \leqq \frac{a+b}{1-d}|(A+\alpha B) x|+\frac{1}{1-d} c(\|x\|),
$$

as $x^{*}$ is an arbitrary element of $A x$. Let $N$ be a positive integer such that

$$
N>\frac{a+b}{1-d}
$$

then

$$
\left\|\frac{1}{N} B x\right\| \leqq \frac{a+b}{(1-d) N}|(A+\alpha B) x|+\frac{1}{(1-d) N} c(\|x\|) .
$$


When $\alpha=0$, we get $A+(1 / N) B$ is maximal monotone; when $\alpha=1 / N$, we get $A+(2 / N) B$ is maximal monotone;...; when $\alpha=(N-1) / N$, we get $A+B$ is maximal monotone, due to Theorem 2.3 in [3].

The following two corollaries allow either $a=1$ or $b=1$.

Corollary 1. In Proposition 1, if $b=1$ and also $\|B x\| \leqq M|A x|+e(\|x\|)(x \in D(A))$, where $M \geqq 0$ is a constant, and $e(r)$ is a nondecreasing function of $r$, then $A+B$ is maximal monotone.

Proof. Let $\varepsilon=(1-a) / 2(M+1)$. For every $x \in D(A)$ and $x^{*} \in A x$, from $(1)(b=1)$ and $\|B x\| \leqq M|A x|+e(\|x\|)$, we have

$$
\begin{aligned}
\|B x\| & \leqq a\left\|x^{*}\right\|+\left\|x^{*}+B x\right\|+c(\|x\|) \\
& \leqq a\left\|x^{*}\right\|+\varepsilon\left\|x^{*}\right\|+\varepsilon\|B x\|+(1-\varepsilon)\left\|x^{*}+B x\right\|+c(\|x\|) \\
& \leqq(a+\varepsilon)\left\|x^{*}\right\|+\varepsilon(M|A x|+e(\|x\|))+(1-\varepsilon)\left\|x^{*}+B x\right\|+c(\|x\|) \\
& \leqq(a+\varepsilon(1+M))\left\|x^{*}\right\|+(1-\varepsilon)\left\|x^{*}+B x\right\|+(c(\|x\|)+\varepsilon e(\|x\|)) .
\end{aligned}
$$

By Proposition 1, the result follows.

Corollary 2. In Proposition 1 , if $a=1$ then $A+\beta B$ is maximal monotone for $0 \leqq \beta<1$.

Proof. For every $x \in D(A)$ and $x^{*} \in D(A)$, from (1) $(a=1)$, we have

$$
\begin{aligned}
\|\beta B x\| & \leqq \beta\left\|x^{*}\right\|+b\left\|\beta x^{*}+\beta B x\right\|+\beta c(\|x\|) \\
& \leqq \beta\left\|x^{*}\right\|+b\left\|(1-\beta) x^{*}\right\|+b\left\|x^{*}+\beta B x\right\|+c(\|x\|) \\
& =(\beta+b(1-\beta))\left\|x^{*}\right\|+b\left\|x^{*}+\beta B x\right\|+c(\|x\|) .
\end{aligned}
$$

As $0 \leqq \beta<1$, the result follows from Proposition 1 .

The following two theorems give the main results in this paper, and establish conditions under which the sum of two maximal monotone operators is maximal monotone.

Theorem 1. Under the conditions of Proposition 1, if inequality (1) (or (2)) is replaced by

$$
\|B x\| \leqq k(\|x\|)|A x|+d|(A+B) x|+c(\|x\|), \text { for every } x \in D(A)
$$


where $0 \leqq k(r)<1$ and $c(r) \geqq 0$ are nondecreasing functions of $r$, and $0 \leqq d<1$ is a constant, then $A+B$ is maximal monotone.

Proof. Without loss of generality, we may assume that $0 \in D(A), 0 \in A 0$ and $0 \in B 0$. This can be achieved by shifting the domain and the range of $A$ and $B$. For every $a>1$, there exists a strictly convex equivalent norm \|\|$_{a}$ on $X$ such that \|\|$_{a}^{*}$ on $X^{*}$ is strictly convex and equivalent to \|\|$^{*}$ and $a^{-1}\|x\| \leqq\|x\|_{a} \leqq a\|x\|, a^{-1}\left\|x^{*}\right\|^{*} \leqq\left\|x^{*}\right\| * \leqq a\left\|x^{*}\right\|^{*}$, for all $x \in X, x^{*} \in X^{*}$ (see Theorem 1.1 in [3]). It is well-known that if for every $f^{*} \in X^{*}$ and $u \in X$, there exists an $a>1$ such that $f^{*}+F_{a}(u) \in R\left(F_{a}+A+B\right)$, where $F_{a}$ is the duality mapping corresponding to \|\|$_{a}$, then $A+B$ is maximal montone $([3$, Lemma 1.1]). This can be shown by considering solutions of the equation

$$
F_{a}\left(x_{\lambda}\right)+x_{\lambda}^{*}+B_{\lambda}^{a} x_{\lambda}=f^{*}+F_{a}(u), x_{\lambda} \in D(A), x_{\lambda}^{*} \in A x_{\lambda},
$$

where $B_{\lambda}^{a}$ and $J_{\lambda}^{a}$ are defined as above but using the duality mapping $F_{a}$ in place of $F$. For every $f^{*} \in X^{*}, u \in X$ and fixed $a$, this equation has a unique solution $x_{\lambda}$ (see [5]). If for some fixed $a,\left\{\left\|B_{\lambda}^{a} x_{\lambda}\right\|_{a}\right\}$ is bounded as $\lambda$ tends to zero, then $f^{*}+$ $F_{a}(u) \in R\left(F_{a}+A+B\right)([3$, Theorem 2.1]). From (3) we have

$$
\|B x\| \leqq|A x|+d|(A+B) x|+c(\|x\|) .
$$

Hence for every $0<\lambda<1, A+(1-\lambda) B$ is maximal monotone due to Corollary 2. It follows that, for any fixed $a>1$, there exists $y_{\lambda} \in D(A), y_{\lambda}^{*} \in A y_{\lambda}$, such that

$$
F_{a}\left(y_{\lambda}\right)+y_{\lambda}^{*}+(1-\lambda) B y_{\lambda}=f^{*}+F_{a}(u) \text {. }
$$

So we have

$$
F_{a}\left(y_{\lambda}\right)-F_{a}\left(x_{\lambda}\right)+y_{\lambda}^{*}-x_{\lambda}^{*}+(1-\lambda) B y_{\lambda}-B_{\lambda}^{a} x_{\lambda}=0
$$

due to (4) and (5). Multiplying (6) by $y_{\lambda}-x_{\lambda}$ yields

$$
\left((1-\lambda) B y_{\lambda}-B_{\lambda}^{a} x_{\lambda}, y_{\lambda}-x_{\lambda}\right) \leqq 0
$$

since $F_{a}$ and $A$ are monotone. This can be written

$$
\left(B y_{\lambda}-B_{\lambda}^{a} x_{\lambda}, y_{\lambda}-J_{\lambda}^{a} x_{\lambda}\right)-\lambda\left(B y_{\lambda}-B_{\lambda}^{a} x_{\lambda}, F_{a}^{-1}\left(B_{\lambda}^{a} x_{\lambda}\right)\right)-\lambda\left(B y_{\lambda}, y_{\lambda}-x_{\lambda}\right) \leqq 0,
$$

since $x_{\lambda}=J_{\lambda}^{a} x_{\lambda}+\lambda F_{a}^{-1}\left(B_{\lambda}^{a} x_{\lambda}\right)$. Because $B$ is monotone and $B_{\lambda}^{a} x_{\lambda} \in B J_{\lambda}^{a} x_{\lambda}$, this gives $\left(B_{\lambda}^{a} x_{\lambda}-B y_{\lambda}, F_{a}^{-1}\left(B_{\lambda}^{a} x_{\lambda}\right)\right) \leqq\left(B y_{\lambda}, y_{\lambda}-x_{\lambda}\right)$. Hence

$$
\begin{aligned}
\left\|B_{\lambda}^{a} x_{\lambda}\right\|_{a}^{2} & \leqq\left(B y_{\lambda}, F_{a}^{-1}\left(B_{\lambda}^{a} x_{\lambda}\right)\right)+\left(B y_{\lambda}, y_{\lambda}-x_{\lambda}\right) \\
& \leqq\left\|B y_{\lambda}\right\|_{a}\left\|B_{\lambda}^{a} x_{\lambda}\right\|_{a}+\left\|B y_{\lambda}\right\|_{a}\left(\left\|y_{\lambda}\right\|_{a}+\left\|x_{\lambda}\right\|_{a}\right) .
\end{aligned}
$$


Multiplying (4) by $x_{\lambda}$ yields

$$
\left\|x_{\lambda}\right\|_{a} \leqq\left\|f^{*}\right\|_{a}+\|u\|_{a} \leqq a\left(\left\|f^{*}\right\|+\|u\|\right)
$$

since $B_{\lambda}^{a} 0=0$. Multiplying (5) by $y_{\lambda}$ yields

$$
\left\|y_{\lambda}\right\|_{a} \leqq\left\|f^{*}\right\|_{a}+\|u\|_{a} \leqq a\left(\left\|f^{*}\right\|+\|u\|\right)
$$

Let $R=2\left(\left\|f^{*}\right\|+\|u\|\right)$ and choose an $a$ such that $1<a<2$, and $a^{2} e<1$, where $e=$ $\max (k(R), d)$. From (3) and (5) we get

$$
\begin{aligned}
&\left\|B y_{\lambda}\right\|_{a} \leqq a\left\|B y_{\lambda}\right\| \\
& \leqq a k(R)\left|A y_{\lambda}\right|+a d\left|(A+B) y_{\lambda}\right|+a c(R) \\
& \leqq a k(R)\left\|y_{\lambda}^{*}\right\|+a d\left\|y_{\lambda}^{*}+B y_{\lambda}\right\|+a c(R) \\
& \leqq a k(R)(1-\lambda)\left\|B y_{\lambda}\right\|+a k(R)\left(\left\|F_{a}\left(y_{\lambda}\right)\right\|+\left\|f^{*}\right\|+\left\|F_{a}(u)\right\|\right) \\
&+a d \lambda\left\|B y_{\lambda}\right\|+a d\left(\left\|F_{a}\left(y_{\lambda}\right)\right\|+\left\|f^{*}\right\|+\left\|F_{a}(u)\right\|\right)+a c(R) \\
& \leqq a^{2} e\left\|B y_{\lambda}\right\|_{a}+2 c(R)+16 R .
\end{aligned}
$$

So $\left\|B y_{\lambda}\right\|_{a} \leqq M_{0}$, where

$$
M_{0}=\frac{2(c(R)+8 R)}{1-a^{2} e}
$$

Hence, using (7) we obtain

$$
\left\|B_{\lambda}^{a} x_{\lambda}\right\|_{a}^{2} \leqq M_{0}\left\|B_{\lambda}^{a} x_{\lambda}\right\|_{a}+2 R M_{0}
$$

So $\left\{\left\|B_{\lambda}^{a} x_{\lambda}\right\|_{a}\right\}$ is bounded and the proof is completed.

Proposition 2. Let $X$ be a reflexive Banach space with $X$ and $X^{*}$ strictly convex, $A$ and $B$ be maximal monotone operators from $X$ to $X^{*}$, with $B$ single-valued and $D(A) \subseteq D(B)$. If

$$
\left(F^{-1} B x, x^{*}\right) \geqq-k\left\|x^{*}\right\|^{2}-c(\|x\|)\left\|x^{*}\right\|-d(\|x\|), \text { for every } x \in D(A), x^{*} \in A x,
$$

and

$$
\|B x\| \leqq M|A x|+e(\|x\|), \quad \text { for every } x \in D(A)
$$


where $0 \leqq k<1$ and $M \geqq 0$ are constants, and $c(r) \geqq 0, d(r) \geqq 0$ and $e(r) \geqq 0$ are nondecreasing functions of $r$, then $A+B$ is maximal monotone.

Proof. From (8), we have

$$
\begin{aligned}
\|B x\|^{2} & \leqq\left(F^{-1} B x, x^{*}+B x\right)+k\left\|x^{*}\right\|^{2}+c(\|x\|)\left\|x^{*}\right\|+d(\|x\|) \\
& \leqq\|B x\|\left\|x^{*}+B x\right\|+k\left\|x^{*}\right\|^{2}+c(\|x\|)\left\|x^{*}\right\|+d(\|x\|) .
\end{aligned}
$$

So

$$
\begin{aligned}
\left(\|B x\|-\frac{1}{2}\left\|x^{*}+B x\right\|\right)^{2} & \leqq \frac{1}{4}\left\|x^{*}+B x\right\|^{2}+k\left\|x^{*}\right\|^{2}+c(\|x\|)\left\|x^{*}\right\|+d(\|x\|) \\
& \leqq\left(\frac{1}{2}\left\|x^{*}+B x\right\|+\sqrt{k}\left\|x^{*}\right\|+\frac{1}{\sqrt{k}} c(\|x\|)+\sqrt{d(\|x\|)}\right)^{2} .
\end{aligned}
$$

That is,

$$
\|B x\| \leqq\left\|x^{*}+B x\right\|+\sqrt{k}\left\|x^{*}\right\|+\frac{1}{\sqrt{k}} c(\|x\|)+\sqrt{d(\|x\|)}
$$

By Corollary 1, the result follows.

Theorem 2. Suppose the conditions of Proposition 2 hold, with (8) replaced by

$$
\begin{aligned}
\left(F^{-1} B x, x^{*}\right) \geqq & -k(\|x\|)\left\|x^{*}\right\|^{2}-c\left(\left\|x^{*}\right\|\right) \\
& -h(\|x\|) p\left(\left\|x^{*}\right\|\right), \text { for every } x \in D(A), x^{*} \in A x,
\end{aligned}
$$

where $0 \leqq k(r)<1, c(r) \geqq 0, h(r) \geqq 0$ and $p(r) \geqq 0$ are nondecreasing functions of $r, c(r) / r^{2} \rightarrow 0$ as $r \rightarrow \infty$, and $p(r) / r$ is bounded when $r \rightarrow \infty$. Then $A+B$ is maximal monotone.

Proof. For every $f^{*} \in X^{*}$, there exists $x_{\lambda} \in D(A), x_{\lambda}^{*} \in A x_{\lambda}$, such that

$$
F\left(x_{\lambda}\right)+x_{\lambda}^{*}+B_{\lambda} x_{\lambda}=f^{*} .
$$

We only need prove that $\left\{\left\|B_{\lambda} x_{\lambda}\right\|\right\}$ is bounded when $\lambda \rightarrow 0$.

For every $0<\delta<1$, from (9), we have

$$
\left(F^{-1}(\delta B x), x^{*}\right) \geqq-\delta\left\|x^{*}\right\|^{2}-c\left(\left\|x^{*}\right\|\right)-h(\|x\|) p\left(\left\|x^{*}\right\|\right) .
$$


Since $c(r) / r^{2} \rightarrow 0$ as $r \rightarrow \infty$ there exists $r_{0}$ such that $c(r) / r^{2}<(1-\delta) / 2$ for $r>r_{0}$. Therefore

$$
c\left(\left\|x^{*}\right\|\right) \leqq \frac{1-\delta}{2}\left\|x^{*}\right\|^{2}+c\left(r_{0}\right)
$$

Since $p(r) / r$ is bounded as $r \rightarrow \infty$, there exists $r_{1} \geqq 0$ and $M_{1} \geqq 0$ such that $p(r) / r \leqq M_{1}$, when $r>r_{1}$. So we have $p\left(\left\|x^{*}\right\|\right) \leqq p\left(r_{1}\right)+M_{1}\left\|x^{*}\right\|$. Hence

$$
\left(F^{-1}(\delta B x), x^{*}\right) \geqq-\frac{1+\delta}{2}\left\|x^{*}\right\|^{2}-M_{1} h(\|x\|)\left\|x^{*}\right\|-\left(c\left(r_{0}\right)+p\left(r_{1}\right) h(\|x\|)\right) .
$$

Therefore $A+\delta B$ is maximal monotone by Proposition 2 . For every $\lambda, 0<\lambda<1$, $A+(1-\lambda) B$ is maximal monotone, so there exists $y_{\lambda} \in D(A), y_{\lambda}^{*} \in A y_{\lambda}$, such that

$$
F\left(y_{\lambda}\right)+y_{\lambda}^{*}+(1-\lambda) B y_{\lambda}=f^{*} \text {. }
$$

Without loss of generality we may assume that $0 \in D(A), 0 \in A 0$ and $0 \in B 0$. Similarly to the proof of Theorem 1, we obtain

$$
\left\|B_{\lambda} x_{\lambda}\right\|^{2} \leqq\left\|B y_{\lambda}\right\|\left(\left\|x_{\lambda}\right\|+\left\|y_{\lambda}\right\|\right)+\left\|B y_{\lambda}\right\|\left\|B_{\lambda} x_{\lambda}\right\|
$$

and

$$
\left\|x_{\lambda}\right\| \leqq\left\|f^{*}\right\|,\left\|y_{\lambda}\right\| \leqq\left\|f^{*}\right\| .
$$

From (9), (11) and (13), we have

$$
\begin{aligned}
\left\|y_{\lambda}^{*}\right\|^{2} \leqq & \left(2\left\|f^{*}\right\|+(1-\lambda)\left\|B y_{\lambda}\right\|\right)^{2} \\
= & (1-\lambda)^{2}\left\|B y_{\lambda}\right\|^{2}+4(1-\lambda)\left\|f^{*}\right\|\left\|B y_{\lambda}\right\|+4\left\|f^{*}\right\|^{2} \\
\leqq & (1-\lambda)\left(F^{-1} B y_{\lambda},(1-\lambda) B y_{\lambda}\right)+4\left\|f^{*}\right\|\left(\left\|y_{\lambda}^{*}\right\|+2\left\|f^{*}\right\|\right)+4\left\|f^{*}\right\|^{2} \\
= & (1-\lambda)\left(F^{-1} B y_{\lambda}, f^{*}-F\left(y_{\lambda}\right)\right)-(1-\lambda)\left(F^{-1} B y_{\lambda}, y_{\lambda}^{*}\right) \\
& +4\left\|f^{*}\right\|\left\|y_{\lambda}^{*}\right\|+12\left\|f^{*}\right\|^{2} \\
\leqq & 2\left\|f^{*}\right\|\left\|(1-\lambda) B y_{\lambda}\right\|+k\left(\left\|f^{*}\right\|\right)\left\|y_{\lambda}^{*}\right\|^{2}+c\left(\left\|y_{\lambda}^{*}\right\|\right)+h\left(\left\|f^{*}\right\|\right) p\left(\left\|y_{\lambda}^{*}\right\|\right) \\
& +4\left\|f^{*}\right\|\left\|y_{\lambda}^{*}\right\|+12\left\|f^{*}\right\|^{2} \\
\leqq & k\left(\left\|f^{*}\right\|\right)\left\|y_{\lambda}^{*}\right\|^{2}+c\left(\left\|y_{\lambda}^{*}\right\|\right)+h\left(\left\|f^{*}\right\|\right) p\left(\left\|y_{\lambda}^{*}\right\|\right)+6\left\|f^{*}\right\|\left\|y_{\lambda}^{*}\right\|+16\left\|f^{*}\right\|^{2},
\end{aligned}
$$


which implies that $\left\{\left\|y_{\lambda}^{*}\right\|\right\}$ is bounded as $\lambda \rightarrow 0$. Therefore $\left\{\left\|B y_{\lambda}\right\|\right\}$ is bounded due to (11), and $\left\{\left\|B_{\lambda} x_{\lambda}\right\|\right\}$ is bounded due to (12).

Remark 1. Our Theorems 1 and 2 generalize Theorems 2.3 in [3], although we suppose that $B$ is single-valued.

Remark 2. We can obtain corresponding results for $m$-accretive operators as follows: Let $X$ be a Banach space, with its dual $X^{*}$ uniformly convex, and let $A$ and $B$ be $m$ accretive operators in $X$, with $B$ single-valued and with $D(A) \subseteq D(B)$. If either (3) or

$$
(y, F(B x)) \geqq-k(\|x\|)\|y\|^{2}-c(\|y\|)-h(\|x\|) p(\|y\|), \quad \text { for every } x \in D(A), y \in A x,
$$

(notice the similarity to (9)) and

$$
\|B x\| \leqq M|A x|+e(\|x\|)
$$

hold, where $k(r), c(r), p(r), e(r), M$ are the same as that in Theorem 2 , then $A+B$ is $m$-accretive. If (1) holds when $b=1$, and $\|B x\| \leqq M|A x|+e(\|x\|)$, then $A+B$ is $m$-accretive.

Details concerning accretive operators and known results needed for the proof can be found in Section 3 of Chapter 2 in [2] (e.g. Lemma 3.2 and Theorem 3.5). The method of proof is quite similar to that for the monotone case, so we omit it.

In addition, for the linear case, the condition $\|B x\| \leqq M|A x|+e(\|x\|)$ holds automatically, because both $A$ and $B$ are closed ([2, Proposition 3.4 in Chapter 2]), so $B$ is $A$ bounded (see Theorem 3.3 in Chapter 5 of Goldberg [7]).

\subsection{Ranges of sums of maximal monotone operators}

For two subsets $E$ and $F$ of $X$ or $X^{*}$, we write $E \simeq F$ to denote that $E$ and $F$ have the same interiors and the same closures. In [4], Brezis-Haraux gave various conditions under which $R(A+B) \simeq R(A)+R(B)$ for monotone operators $A$ and $B$. The proof of our results uses the method of Pazy ([4, Appendix]) and of Reich [9].

Theorem 3. Let $X$ be a reflexive Banach space with $X, X^{*}$ strictly convex, $A$ and $B$ be maximal monotone operators from $X$ to $X^{*}$, and $B$ be single-valued with $D(A) \subseteq D(B)$. If

$$
\|B x\| \leqq a|A x|+b|(A+B) x|+c, \quad \text { for every } x \in D(A),
$$

where $0 \leqq a<1,0 \leqq b<1$, and $c \geqq 0$ are constants, then $R(A)+R(B) \simeq R(A+B)$.

Proof. By Theorem $1, A+B$ is maximal monotone. Therefore, for every $f^{*} \in X^{*}$ and $\lambda>0$, there exists $x_{\lambda} \in D(A), x_{\lambda}^{*} \in A x_{\lambda}$, such that

$$
\lambda F x_{\lambda}+x_{\lambda}^{*}+B x_{\lambda}=f^{*} .
$$


If for every $f^{*} \in R(A)+R(B),\left\{x_{\lambda}^{*}\right\}$ is bounded as $\lambda \rightarrow 0$, then $R(A)+R(B) \simeq R(A+B)$ ([9, Proposition 2.3]). For any fixed $x_{0} \in D(A)$, let $y_{0}^{*} \in A x_{0}$. Multiplying (15) by $x_{\lambda}-x_{0}$ and using the monotonicity of $A$ and $B$, we get

$$
\lambda\left\|x_{\lambda}\right\|^{2} \leqq \lambda\left\|x_{0}\right\|\left\|x_{\lambda}\right\|+\left(\left\|y_{0}^{*}\right\|+\left\|B x_{0}\right\|+\left\|f^{*}\right\|\right)\left(\left\|x_{\lambda}\right\|+\left\|x_{0}\right\|\right) .
$$

Multiplying by $\lambda$ shows that $\left\{\lambda\left\|x_{\lambda}\right\|\right\}$ is bounded as $\lambda \rightarrow 0$. From (14) and (15) we have

$$
\begin{aligned}
\left\|B x_{\lambda}\right\| & \leqq a\left\|x_{\lambda}^{*}\right\|+b\left\|x_{\lambda}^{*}+B x_{\lambda}\right\|+c \\
& \leqq a\left\|B x_{\lambda}\right\|+(a+b)\left(\left\|f^{*}\right\|+\lambda\left\|x_{\lambda}\right\|\right)+c .
\end{aligned}
$$

So $\left\{B x_{\lambda}\right\}$ is bounded as $\lambda \rightarrow 0$. As a result, $\left\{x_{\lambda}^{*}\right\}$ is bounded as $\lambda \rightarrow 0$ due to (15).

Theorem 4. Under the conditions of Theorem 3, if (14) is replaced by

$$
\left(F^{-1} B x, x^{*}\right) \geqq-k\left\|x^{*}\right\|^{2}-c\left\|x^{*}\right\|-d, \text { for every } x \in D(A), x^{*} \in A x
$$

and

$$
\|B x\| \leqq M|A x|+e, \text { for every } x \in D(A),
$$

where $0 \leqq k<1, c \geqq 0, d \geqq 0, M \geqq 0$ and $e \geqq 0$ are constants, then $R(A)+R(B) \simeq R(A+B)$.

Proof. Similarly to the proof of Proposition 2 and Corollary 1, we can prove that (16) and (17) implies (14), so the conclusion is immediate.

\section{REFERENCES}

1. H. Aтtouch, On the maximality of the sum of two maximal monotone operators. Nonlinear Anal., Theory, Methods \& Appl. 5 (1981), 143-147.

2. V. BARBU, Nonlinear Semigroups and Differential Equations in Banach Spaces (Nordhoff International Publishing, Leyden, The Netherlands, 1976).

3. H. Brezis, M. G. Crandall and A. Pazy, Perturbations of nonlinear maximal monotone sets in Banach space. Comm. Pure Appl. Math. 23 (1970), 123-144.

4. H. Brezis and A. Haraux, Image d'une somme d'operateurs monotones et applications, Israel J. Math. 23 (1976), 165-186.

5. F. E. Browder, Nonlinear maximal monotone operators in Banach space, Math. Ann. 175 (1968), 89-113.

6. B. Calvert, Maximal monotonicity and $m$-accretivity of $A+B$. Atti. Accad. Naz. Lincei. Rend. Cl. Sci. Fis. Mat. Naturali (8) 49 (1970), 357-363.

7. S. Goldberg, Unbounded Linear Operators: Theory and Application-Series in Higher Math. (McGraw-Hill, New York, 1966). 
8. T. Kato, Accretive operators and nonlinear evolution equations in Banach spaces, in Proc. Symp. Pure Math (Nonlinear Functional Anal.) (American Math. Society, Providence, Rhode Island, 1970), 138-161.

9. S. Reich, The range of sums of accretive and monotone operators, J. Math. Anal. Appl. 68 (1979), 310-317.

10. R. T. Rockafellar, On the maximality of sums of nonlinear monotone operators, Trans. Amer. Math Soc. 149 (1970), 75-88.

Department of Mathematics

UNIVERSITY OF GLASGOW

GLASGOW G12 8QW 